\title{
Interdisciplinary Endoscopy
}

\author{
Chair: \\ Hans-Dieter Allescher ${ }^{\mathrm{a}}$ \\ Participants: Evgeny D. Fedorov ${ }^{b}$ \\ Peter D. Siersema ${ }^{f}$ \\ Jürgen Hochberger ${ }^{c} \quad$ Florian Schreiber $^{d}$ \\ Stefan Seewald ${ }^{\mathrm{e}}$ \\ a Zentrum Innere Medizin - Gastroenterologie, Hepatologie, Stoffwechsel, Nephrologie, Klinikum Garmisch-Partenkirchen, \\ Garmisch-Partenkirchen, Germany; \\ ${ }^{b}$ Department for Abdominal Surgery, Gastroenterology \& Endoscopy, Pirogov RNRMU, Moscow University Hospital N31, Moscow, Russia; \\ ${ }^{c}$ Responsable de la Section de Gastroentérologie et d'Endoscopie digestive, Pôle Hépato-Digestif Interdisciplinaire, Hôpitaux \\ Universitaires de Strasbourg - Nouvel Hôpital Civil et IHU, Strasbourg (Cedex), France; \\ d Klinische Abteilung für Gastroenterologie und Hepatologie, Universitätsklinik für Innere Medizin, Medizinische Universität Graz, Graz, \\ Austria; \\ e GastroZentrum Hirslanden, Klinik Hirslanden, Zürich, Switzerland; \\ ${ }^{f}$ Department of Gastroenterology and Hepatology (route 455), Radboud University Medical Center, Nijmegen, the Netherlands
}

\section{Question 1: How long does the qualification for interventional endoscopy take in your country? Is interventional endoscopy limited to gastroenter- ologists/surgeons? Do you have a special certificate for interventional endoscopy?}

Allescher: In Germany interventional endoscopy is part of the training for being a gastroenterology specialist. Depending on the pathway, it takes either 3 years of general internal medicine training and 3 years of gastroenterology training including interventional endoscopy or 5 years of general internal medicine training and then another 3 years of specialized gastroenterology training. The training standards, especially for more advanced procedures, which can be achieved with this two training pathways differ quite substantially.

The education for visceral surgeons comprised 2 years of basic common training and a 4-year interval of visceral surgery training. This education also includes endoscopy training, but specialized interventional endoscopy is not mandatory. A specialized visceral medicine training including another 3 years of visceral surgery can be added to this education.

In Germany, there is no specialized certificate for interventional endoscopy, neither in gastroenterology nor in surgery.

Fedorov: The qualification for interventional endoscopy in Russia takes 2.5 years.

Interventional endoscopy in our country predominantly is limited to surgeons. Even diagnostic endoscopy, from the very begin- ning at the end of 1960s, has been limited to surgeons for many years, and only during the last 5-7 years gastroenterologists are more and more involved.

In Russia, we have not a special certificate for interventional endoscopy; a certificate for endoscopy incorporates interventional endoscopy. But! The strong tendency is to separate diagnostic (gastroenterological) and interventional ('surgical') endoscopy.

Hochberger: In France, the education for interventional endoscopy takes 4 years of pure training in gastroenterology and hepatology ('interne'). No common trunk in internal medicine. Two additional years ('clinicat') as assistant consultant ('Chef de Clinique' or 'Assistant Clinique') are needed to work in private practice or as specialist at the hospital. Thus education time amounted to 6 years. Endoscopy training is up to now officially designated to gastroenterologists, and not to surgeons. There is up to now no diploma in advanced endoscopy which is, aside from gastric/colonic/ variceal band ligation, mostly limited to the last 2 years (endoscopic ultrasound (EUS), endoscopic retrograde cholangiopancreatography (ERCP), esophageal stents, endoscopic mucosal resection (EMR) etc.).

Of the 4 first years, a minimum of 2 years has to be done in a gastroenterology and hepatology department at a university hospital; the other 2 years can also be done in other specialties and at teaching hospitals. The interns rotate every 6 months and come back a second time to your gastrointestinal unit. Opt-out possibilities are: radiology and ultrasound (have to do even night and week-

\section{KARGER}

Fax +497614520714

\section{(c) 2016 S. Karger GmbH, Freiburg}

$2297-4725 / 16 / 0321-0059 \$ 39.50 / 0$
Prof. Dr. Hans-Dieter Allescher

Zentrum Innere Medizin - Gastroenterologie, Hepatologie, Stoffwechsel, Nephrologie Klinikum Garmisch-Partenkirchen

Auenstraße 6, 82467 Garmisch-Partenkirchen, Germany

Hans-Dieter.Allescher@ klinikum-gap.de 
end duties for CT, MRI, and US after 6 weeks), radiotherapy, intensive care / emergency medicine (not obligatory otherwise), oncology, radiotherapy etc.

These 4 years include an obligatory thesis (thèse en médecine) and a scientific work ('mémoire'). Both are presented at conferences of internes and their university professors twice a year organized for the major areas (for 5-6 universities with a total of 50 internes and 10-12 professors) and have to be defended in front of a committee.

Schreiber: As there is no certificate for interventional endoscopy in Austria, it is limited (from the side of reimbursement) to gastroenterologists and surgeons. There is no distinct time frame for trainees to accomplish the authorization (characterized only by reimbursement), but a minimum count for some interventions.

Seewald: In Switzerland, interventional endoscopy is performed by gastroenterologists. There is no specific qualification for 'interventional endoscopy'. However, to ensure quality of ERCP, the Swiss Society for Gastroenterology provides a specific certificate for ERCP. 100 ERCPs, 50 papillotomies, 25 stone extractions and 25 drainages are required. Every 3 years recertification is needed.

Siersema: a) In the Netherlands, we are still developing, but in the new training manual which needs to be followed by all units, we plan to select gastroenterologists that are still in training in the final 1-2 years to learn therapeutic endoscopy. We do however realize that this is not enough, and therefore we are planning to have an additional 1-2 years of fellowships in advanced endoscopy. b) Therapeutic endoscopy is fortunately still limited to gastroenterologists. c) As of now, we do not have a certificate but you can gain 'competency' in procedures.

\section{Question 2: How is the interface between clinical and ambulatory setting organized? Is interventional endoscopy also performed in private practice or in outpatient settings? Up to which level (ERCP, EUS, endoscopic submucosal dissection (ESD)) is interventional endoscopy offered in an outpatient setting?}

Allescher: Usually there is a relatively strict separation between endoscopists working in the clinical setting and private practice. Private practice is usually run in a private office with sole funding through the fees obtained from insurance companies based on the procedures performed. In most private offices, interventional endoscopy, which requires postprocedural surveillance is not performed as the reimbursement for these procedures is not really sufficiently established in a detailed fashion and thus is not cost efficient. Additionally the risks with regard to time consumption and the difficulties with planning and efficient time management make interventional endoscopy relatively unattractive for private practice. In some instances private endoscopy units collaborate either with or are attached to a clinic, which could be an attractive model for the future.

Fedorov: The interface between clinical and ambulatory setting is organized relatively simply. Diagnostic procedures are mostly done in ambulatory settings and interventional procedures in clinical settings. It is allowed to perform diagnostic (ambulatory) procedures in the clinical setting, but not vice versa.

Interventional endoscopy is more and more performed in private practice (which means private hospitals, and not private offices). It is still not common, or even not allowed, to perform interventional endoscopy in outpatient settings without available surgical department and an operating room ready to use. But another strong tendency of the last years, particularly in private practice, is to reduce patients' length of stay to about a few hours, instead of a few days.

As far as I know the situation in Russia, only diagnostic EUS is offered in an outpatient setting. ERCP, interventional EUS, and ESD are offered almost exclusively in inpatient settings. There were some exceptions for relatively 'simple' ERCP or ESD, but anyway these outpatient facilities have a strong connection with a clinic or a surgical department.

Hochberger: Many gastroenterologists working in private practice are at the same time affiliated to a 'private hospital'. Private means also regularly insured patients but a private hospital organization in contrast to the public health service.

There is no official data on outpatient treatment for interventional endoscopy. To do interventional endoscopy in a hospital or private clinic environment with short-term hospitalization seems to be more common in France than in Germany or Switzerland.

Schreiber: There is no general regulation where or in which setup interventional procedures can be performed. Usually only simple polypectomies are done in an outpatient setting.

But theoretically every procedure could be performed in an outpatient setting, if the whole procedure is done according to the specific guidelines and if there is an agreement with the social insurance carrier or a contract with the payer concerning reimbursement.

Seewald: Interventional endoscopy is also performed in private practice or in outpatient settings. EUS is regularly performed in an outpatient setting, whereas ERCP and ESD are performed in the hospital. There are a number of private practices at hospitals or affiliated to hospitals with gastroenterologists as inpatient physicians'.

Siersema: In the Netherlands, we do not have a real private practice; endoscopy is performed in hospitals, and therapeutic endoscopy is performed at different 'severity' levels. 


\section{Question 3: Do specialists from the clinic work in outpatient settings?}

Allescher: In the past this was very rare. In the meantime the borders between clinic and private practice become more permeable. Doctors from the clinic can work part time in private practice or in a so-called MVZ - Medizinisches Versorgungszentrum which is comparable to a specialist center.

Fedorov: Yes, specialists from the clinic often work (as part work) in outpatient settings; it is the common practice of the last years, mostly due to financial circumstances.

Hochberger: Yes and vice versa. We have 5 gastroenterologists - one every day of the week - who do endoscopies (gastroscopies, colonoscopies) in the morning for $2-3 \mathrm{~h}$ together with our interns.

Schreiber: Yes, specialists with clinical background are allowed to work in a private setting - strictly - during their leisure time. This has been more and more restricted during the last years in most countries/ clinics and to date depends on approval by the board (conflict of interests etc.).

Seewald: Yes.

Siersema: No, all gastroenterologists work in a hospital setting. The only exception is outpatient setting for screening colonoscopy.

\section{Question 4: How is the interaction with other specialists such as ENT (ear, nose and throat) specialists, pulmonologists, or radiologists organized?}

Allescher: In Germany ENT specialists do rigid and flexible endoscopy of the larynx and the pharynx and in the clinical setting they sometime perform rigid esophagoscopy. There are several diseases like reflux-induced change of the larynx and paranasal sinuses where closed interaction is required. Pulmonologists, who carry out the majority of the bronchoscopies work together with gastrointestinal endoscopists in an interdisciplinary endoscopy unit. In private practice these specialists mostly work separately. Swallow endoscopies (fiber-optic evaluation of swallowing; FEES) are performed by ENT doctors, gastroenterologists, neurologists, and swallow therapists. The interaction with radiologists involves cinematographies on the one side and percutaneous transhepatic cholangiography drainage (PTCD) and cholangioscopies on the other. In many centers, percutaneous transhepatic cholangioscopy (PTCS) and cholangioscopies are performed by gastroenterologists, whereas radiologists do interventional procedures mostly without cholangioscopy.

Fedorov: The interaction with other specialists is organized via their consultations or joint consultations in the most difficult cases.
Sometimes we are working together in the endoscopy room, for example during rendezvous biliary procedures.

Hochberger: ENT specialists do rigid esophagoscopies and dilations. We have a gastrointestinal endoscopy unit which has a common endoscope reprocessing unit with the pneumonologists who are just aside.

Schreiber: Interdisciplinary interaction concerning standard procedures (in my institution - Medical University Graz) is based on individual case conferences. Concerning malignant entities and associated interventions, interdisciplinary collaboration is based on specific tumor boards according to all underlying regulations.

Seewald: Patients with a cancer disease have to be presented at an oncological board. Specific meetings are organized by the societies.

Siersema: In general, there is good collaboration as we all depend on each other every now and then (pulmonology, ENT) or very frequently (intervention, radiology).

\section{Question 5: How are new interventional methods (ESD, peroral endoscopic myotomy (POEM), full-thickness resection device (FTRD), endoscopic necrosectomy) introduced in endoscopy, and how is the reimbursement organized during this introduction?}

Allescher: It is difficult in Germany to get new interventional methods reimbursed. One possibility is to establish an OPS (Operationen- und Prozedurenschlüssel) for an endoscopic procedure which will eventually lead to an DRG with subsequent permanent reimbursement. Another way which can lead to time-limited reimbursement involves the status of so-called NUB (Neue Untersuchungs- und Behandlungsverfahren). Using the NUB, an individual reimbursement for a new procedure at a given institution can be can applied for 1 year. Both procedures are relatively time-consuming and have a latency of at least 1 to several years. In this period no direct reimbursement is possible.

Fedorov: To be precise, FTRD at the moment is not available in our country. The way for introduction of new interventional methods in endoscopy is more or less similar: initial information; texts, pictures, video, life-course etc.; dry lab, then (preferably) wet lab; invited expert; own experience, at the beginning preferably under supervision.

To our great regret there is no special reimbursement for new interventional methods, except private setting. Of course, the hospital pays for the initial instruments or set of instruments, but this is done from time to time without any regular reimbursement system. 
Hochberger: None of those are currently reimbursed as separate exams. Equivalent surgical codes are accepted for certain procedures.

Schreiber: Concerning reimbursement, EMR/ESD and Barrett's radiofrequency ablation (RFA) had become subject to this during the last years. To date POEM or FTRD do not have any specific reward but will have during next months.

Seewald: New interventional methods are introduced by meetings organized by the Swiss Society of Gastroenterology and by individual activities of the interventional endoscopists. In public insurance there is a DRG system for treatments in hospitals, mostly with payment by diagnosis. For instance, ESD is not specifically reimbursed for patients with public insurance. If colonic ESD has been performed and no cancer has been found, reimbursement is much lower than if a cancer has been found. High costs for equipment (knifes, RFA balloons, spy glass etc.) are a problem. There is only an additional fixed reimbursement for metallic stents. In the private sector a reimbursement system has been just established between particular hospitals and a few insurance companies with specific reimbursement of complex endoscopic procedures.

Siersema: a) These procedures are usually performed in specialist therapeutic centers, like ours, AMC, Erasmus etc. b) Reimbursement is not well organized and is now slowly starting to be established on a national level.

\section{Participants}

Prof. Dr. Hans-Dieter Allescher

Zentrum Innere Medizin

Gastroenterologie, Hepatologie, Stoffwechsel, Nephrologie

Klinikum Garmisch-Partenkirchen

Auenstraße 6, 82467 Garmisch-Partenkirchen, Germany

Hans-Dieter.Allescher@klinikum-gap.de

Prof. Dr. Evgeny D. Fedorov

Department for Abdominal Surgery, Gastroenterology \& Endoscopy Pirogov RNRMU, Moscow University Hospital N31, Moscow, Russia efedo@mail.ru

Prof. Dr. Jürgen Hochberger, PU-PH, FASGE

Responsable de la Section de Gastroentérologie et d'Endoscopie digestive, Pôle Hépato-Digestif Interdisciplinaire

Hôpitaux Universitaires de Strasbourg - Nouvel Hôpital Civil et IHU

1 Place de l'Hôpital - BP 426, 67091 Strasbourg (Cedex), France

juehochber@mac.com

Univ. Prof. Dr. Florian Schreiber

Klinische Abteilung für Gastroenterologie und Hepatologie

Universitätsklinik für Innere Medizin, Medizinische Universität Graz

Auenbruggerplatz 15, 8036 Graz, Austria

florian.schreiber@medunigraz.at

Prof. Dr. Stefan Seewald

GastroZentrum Hirslanden

Klinik Hirslanden

Witellikerstrasse 40, 8032 Zürich, Switzerland

Stefan.Seewald@gastrozentrum.ch

Prof. Dr. Peter D. Siersema

Department of Gastroenterology and Hepatology (route 455)

Radboud University Medical Center

Geert Grooteplein Zuid 10, 6525 GA Nijmegen, the Netherlands

peter.siersema@radboudumc.nl 